\title{
Yair Caro
}

Zero-sum bipartite Ramsey numbers

Czechoslovak Mathematical Journal, Vol. 43 (1993), No. 1, 107-114

Persistent URL: http://dml.cz/dmlcz/128379

\section{Terms of use:}

(C) Institute of Mathematics AS CR, 1993

Institute of Mathematics of the Czech Academy of Sciences provides access to digitized documents strictly for personal use. Each copy of any part of this document must contain these Terms of use.

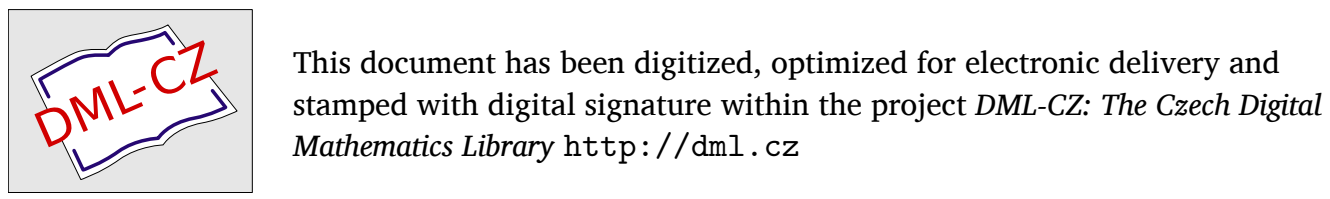




\section{ZERO-SUM BIPARTITE RAMSEY NUMBERS}

YaIr Caro, Oranim

(Received May 2, 1991)

\section{INTRODUCTION}

The starting point of almost all the recent combinatorial research on zero-sum problems is the following theorem.

Theorem A. (Erdös, Ginzburg, Ziv [E(iZ]). Let $m \geqslant k \geqslant 2$ be two integers such that $k \mid m$. Then any sequence of $m+k-1$ integers contains a subsequence of cardinality $m$ the sum of whose elements is divisible by $k$.

There is a rapidly growing literature on zero-sum problems. As can be see in the list of references, most of them dealt with the so-called zero-sum Ramsey numbers, a concept first introduced by Bialostocki and Dierker ([BD1] [BD2]). To describe this concept as well as the bipartite variant we need a few definitions. Let $\mathbb{Z}_{k}$ denote the cyclic additive group of order $k$. A $\mathbb{Z}_{k}$-coloring of the edges of a graph $G_{r}=(V, E)$ is a function $f: E(G) \rightarrow \mathbb{Z}_{k}$. If $\sum_{e \in E(G)} f(e)=0$ (in $\mathbb{Z}_{k}$ ), we say that $G$ is a zero-sum graph $(\bmod k$ ) (with respect to $f$ ). If $k$ divides the number, $e(G)$, of edges of $G$, then the zero-sum Ramsey number $R\left(G, \mathbb{Z}_{k}\right)$ is the smallest integer $t$ such that for every $\mathbb{Z}_{k}$-coloring of $E\left(K_{t}\right)$ there is a zero-sum $(\bmod k)$ copy of $G$ in $K_{t}$.

If $G$ is bipartite and $k \mid e\left(G_{r}\right)$, then the zero-sum bipartite Ramsey number $B\left(G_{r}, \mathbb{Z}_{k}\right)$ is the smallest integer $t$ such that for every $\mathbb{Z}_{k}$-coloring of $E\left(K_{t, t}\right)$ (the complete bipartite graph) there is a zero-sum $(\bmod k)$ copy of $G$ in $K_{t, t}$.

The existence of $B\left(G, \mathbb{Z}_{k}\right)$ follows from the trivial inequality $B\left(G, \mathbb{Z}_{k}\right) \leqslant B(G, k)$, where $B(G, k)$ is the classical bipartite Ramsey number using $k$ colors (see e.g. [GRS]).

The first problem we consider here, in section 2 , is that of estimating $B\left(G, \mathbb{Z}_{2}\right)$. As shown in [ALCA] $R\left(G, \mathbb{Z}_{2}\right) \leqslant|G|+2$.

Define $m(G)=\min \{|A|, V(G)=A \cup B,|A| \geqslant|B|\}$ where the minimum is taken over all the representations of $G$ as a bipartite graph with classes $A$ and $B$, (e.g., $\left.m\left(K_{1, n}\right)=n, m\left(K_{2,3} \cup K_{4.7}\right)=9\right)$. 
We prove that $B\left(G, \mathbb{Z}_{2}\right) \leqslant m(G)+1$ and discuss some exact cases. The second problem we consider here, in Section 3 , is that of estimating $B\left(K_{n, m}, \mathbb{Z}_{k}\right)$. We prove that if $K \mid n(n \geqslant m)$ then $B\left(K_{n, m}, \mathbb{Z}_{k}\right) \leqslant n+k-1$ and explore some cases in which this bound is sharp. In contrast we prove that $B\left(K_{n, n}, \mathbb{Z}_{n^{2}}\right)$ grows exponentially. Essentially the same behaviour is known to hold for $R\left(K_{n}, \mathbb{Z}_{k}\right)$ vs. $R\left(K_{n}, \mathbb{Z}_{\left(\begin{array}{c}n \\ 2\end{array}\right)}\right)$ as proved in [CAR1] [CAR5] [ALCA].

The third problem considered in Section 3 , is to evaluate $B\left(t K_{2}, \mathbb{Z}_{k}\right)$ when $k \mid t$ and $t K_{2}$ is the disjoint union of $t$ edges. Using theorem $A$ and a construction we prove $B\left(t K_{2}, \mathbb{Z}_{k}\right)=t+k-1$. Some related problems will be considered.

We follow the standard notation of [BOLI]. In particular $e\left(G^{\prime}\right)$ denotes the number of edges of $\left(i . S_{n}\right.$ denotes the group of permutations of $n$-element set. $C_{n}$ denotes the cyclic group of permutations of $n$-element set. For a finite set $S$ let

$$
\delta\left(S^{\prime}\right)= \begin{cases}1 & \text { if }|S| \equiv 0(\bmod 2) \\ 0 & \text { if }|S| \equiv 1(\bmod 2) .\end{cases}
$$

\section{AN UPPER BOUND FOR $B\left(G, \mathbb{Z}_{2}\right)$}

The essence of this section can be summarized as:

Theorem 1. Let ( $i$ be a bipartite graph such that $2 \mid e(G)$.

(i) if $m(G) \equiv 1(\bmod 2)$ then $B\left(G, \mathbb{Z}_{2}\right)=m(G)$.

(ii) if $m(G) \equiv 0(\bmod 2)$ then $B\left(G, \mathbb{Z}_{2}\right) \leqslant m(G)+1$.

(iii) if $m(G) \equiv 0(\bmod 2)$ and $A$ realizes $m(G),|A|>|B|$, and for every $x \in A$ $\operatorname{deg} x \equiv 0(\bmod 2)$ then $B\left(G, \mathbb{Z}_{2}\right)=m(G)$.

For the proof we apply a method developed in $[A L C A]$. We need Lemma and the following definition.

Definition. Suppose $H_{1}, H_{2}, \ldots, H_{n}$ is a family of subgraphs of $K_{t, t}$. Then the sum modulo-2 of $H_{1}, \ldots, H_{n}$ denoted by $\oplus \sum_{i=1}^{n} H_{i}$, is the subgraph of $K_{t, t}$ whose edges are all those edges of $K_{t, t}$ belonging to an odd number of $H_{i}$-s.

Observe that this is exactly the sum (in $\mathbb{Z}_{2}$ ) of the vectors corresponding to the $H_{i}$-s, where to each $H_{i}$ is associated the characteristic vector, of length $t^{2}$, of its edges. (Exactly $e(C)$ places are 1 and the others are 0. )

In the case that $\oplus \sum_{i=1}^{n} H_{i}$ is the empty graph we write $\oplus \sum_{i=1}^{n} H_{i}=\underline{0}$.

Lemma. (Parity Lemma.) Let $G$ be a bipartite graph so that $2 \mid e(G)$. Then $B\left(G, \mathbb{Z}_{2}\right)$ is the least integer $t$ such that $K_{t, t}$ contains a family $H_{1}, \ldots, H_{n}$ of subgraphs isomorphic to $G, n$ is odd and $\oplus \sum_{i=1}^{n} H_{i}=\underline{0}$. 
Proof. Let $I_{t}(G)$ be the family of all subgraphs of $K_{t, t}$ isomorphic to $G$. To each member $H \in I_{t}\left(G^{\prime}\right)$ we make correspond an equation with $e(G)$ variables, namely $\sum_{e \in E(H)} x_{e}=1\left(\right.$ in $\left.\mathbb{Z}_{2}\right)$.

This system of equations has no solution if $t \geqslant B\left(G, \mathbb{Z}_{2}\right)$, because in this case a zero-sum ( $\bmod 2)$ copy of $G$ will not satisfy its equation. Hence $B\left(G, \mathbb{Z}_{2}\right)$ is the least such $t$.

Recall a basic result from linear algebra: The system $A x=b$ has no solution iff the Ciaussian elimination procedure results in a row of the form $(0,0,0 \ldots, 0, t)$ where $t \neq 0$ (see e.g. [STE] p. 142-143). We find that the above system has no solution iff there is an odd number of equations whose sum (in $\mathbb{Z}_{2}$ ) gives $\underline{0}=1$, and the Lemma follows.

Proof of Theorem 1. Suppose $f: E\left(K_{t, t}\right) \rightarrow \mathbb{Z}_{2}$ where $t=|A|+\delta(A)$, $|A|=m(G)$. Observe that $t=|A|+\delta(A) \equiv 1(\bmod 2)$.

Fix a copy of $G_{i}$ in $K_{t, t}$, and consider the direct product group $C_{t}^{(1)} \times C_{t}^{(2)}:=H$ acting on $V\left(K_{t, t}\right)$, where $C_{t}^{(1)}$ acts cyclically on one class of $K_{t, t}$ and $C_{t}^{(2)}$ on the other class.

How many copies of $C r$ do we get from the action of $H$ ?

Exactly $t^{2} \equiv 1(\bmod 2)$.

On the other hand as $2 \mid e(G)$ every edge of $E\left(K_{t, t}\right)$ appears in exactly $e(G)$ copies of $G$, under the action of $H$. Hence $\oplus \sum_{\sigma \in H} \sigma(G)=\underline{0},|H|=t^{2} \equiv 1(\bmod 2)$ and by the Parity Lemna $B\left(G, \mathbb{Z}_{2}\right) \leqslant t=|A|+\delta(A),|A|=m(G)$ which completes the proof of parts (i) and (ii).

For part (iii) observe that $m(G)=|A| \geqslant|B|+\delta(B)$, (by assumption). Let $f$ : $E\left(K_{t, t}\right) \rightarrow \mathbb{Z}_{2}$, where $t=m\left(G^{\prime}\right)$ and fix a copy of $K_{t, q}$ in $K_{t, t}$ where $q=|B|+\delta(B)$. In $K_{t, q}$ fix a copy of $G$ in such a way that $A$ is in the class of order $t$ and $B$ in the class of order $q$.

Consider the action of the permutation group $C_{q}$ on the class of order $q$. As $q \equiv 1(\bmod 2)$ we get a family of $q$ copies of $G$. On the other hand consider an edge $e=(x, y) \in E\left(K_{t, q}\right)$, where $x \in A$. Clearly $e$ appears in exactly deg $x$ copies of $G$ under the action of $C_{q}$, but $\operatorname{deg} x \equiv 0(\bmod 2)$ hence $\oplus \sum_{\sigma \in C_{q}} \sigma(G)=\underline{0}, q \equiv 1$ $(\bmod 2)$ and by the parity lemma we are done.

A simple observation [ALCA] states that if $2 \mid\left(\begin{array}{c}n \\ 2\end{array}\right)$ then $R\left(K_{n}, \mathbb{Z}_{2}\right)=n+2$. Here we derive a similar result for the complete bipartite graph $K_{m, n}$ when $2 \mid m n$.

Theorem 2. Let $n \geqslant m \geqslant 1$ be integers. Then

$$
B\left(K_{m, n}, \mathbb{Z}_{2}\right)= \begin{cases}n+1 & \text { if } 2 \mid m, m=n \\ n & \text { if } 2 \mid m, n>m \\ n+1 & \text { if } 2 \mid n \text { and } 2 \nmid m .\end{cases}
$$


Proof. (i) Suppose first $2 \mid m, m=n$. Let $f: E\left(k_{n+1, n+1}\right) \rightarrow \mathbb{Z}$. Take $n$ vertices of one side of $K_{n+1, n+1}$, say $u_{1}, \ldots, u_{n}$ and all the $n+1$ vertices of the other side, say $w_{1}, \ldots, w_{n+1}$.

Define a sequence of $n+1$ integers as follows: for $1 \leqslant i \leqslant n+1, a_{i}=\sum_{j=1}^{n} f\left(u_{i}, u_{j}\right)$. By Theorem $A$ there are $n$ terms whose sum is $0(\bmod 2)$, namely $\sum_{i \in I} a_{i} \equiv 0$ $(\bmod 2),|I|=n$. Now $u_{1}, \ldots, u_{n}$ and $\left\{w_{i}, i \in I\right\}$ form a zero-sum copy of $K_{n, n}$. Hence $B\left(K_{n, n}, \mathbb{Z}_{2}\right) \leqslant n+1$. For the lower bound consider $K_{n, n}$ with classes $A=\left\{u_{1}, \ldots, u_{n}\right\}$ and $B=\left\{w_{1}, \ldots, w_{n}\right\}$. Define $f: E\left(K_{n, n}\right) \rightarrow \mathbb{Z}_{2}$ by

$$
f\left(u_{i}, w_{j}\right)= \begin{cases}1 & i=j=1 \\ 0 & \text { otherwise. }\end{cases}
$$

This $\mathbb{Z}_{2}$-coloring implies $B\left(K_{n, n}, \mathbb{Z}_{2}\right)>n$, hence $B\left(K_{n, n}, \mathbb{Z}_{2}\right)=n+1$.

(ii) Suppose $2 \mid m, n>m$. Repeat the argument above for $f: E\left(K_{n, n}\right)-\mathbb{Z}_{\text {? }}$ obtain, in exactly the same way, $B\left(K_{m, n}, \mathbb{Z}_{2}\right) \leqslant n$ and clearly $B\left(K_{m, n}, \mathbb{Z}_{2}\right) \geqslant n$, hence $B\left(K_{m, n}, \mathbb{Z}_{2}\right)=n$.

(iii) Suppose $2 \mid n, n>m$ and $2 \nmid m$. For the upper bound repeat the argument of (i) to obtain $B\left(K_{m, n}, \mathbb{Z}_{2}\right) \leqslant n+1$.

For the lower bound consider $K_{n, n}$ with classes $A=\left\{u_{1}, \ldots, u_{n}\right\}, B=\left\{w_{1}, \ldots, w_{n}\right\}$ and define $f: E\left(K_{n, n}\right) \rightarrow \mathbb{Z}_{2}$ by

$$
f\left(u_{i}, w_{j}\right)= \begin{cases}1 & i=j \\ 0 & \text { otherwise. }\end{cases}
$$

Clearly no zero-sum copy of $K_{n, m}$ exists because for every such copy $H, \sum_{e \in E^{\prime}(H)} f(e)=$ $m \equiv 1(\bmod 2)$. Hence $B\left(K_{m, n}, \mathbb{Z}_{2}\right)=n+1$ completing the proof.

\section{Estimations of $B\left(K_{m, n}, \mathbb{Z}_{k}\right)$}

Let's first extend the argument used in the proof of theorem 2 to investigate $B\left(K_{m, n}, \mathbb{Z}_{k}\right)$ where $k \mid m$ or $k \mid n$.

Theorem 3. Let $n \geqslant m \geqslant 1$ be integers. Then

$$
B\left(K_{m, n}, \mathbb{Z}_{k}\right) \leqslant \begin{cases}m+k-1 & \text { if } k \mid m, m \leqslant n \leqslant m+k-2 \\ n & \text { if } k \mid m, n \geqslant m+k-1 \\ n+k-1 & \text { if } k \mid n \text { and } k \nmid m .\end{cases}
$$


(ii) put $f(k)=\left\{\begin{array}{ll}k-1 & \text { if } k \text { is a prime } \\ \lfloor\sqrt{k-1}\rfloor & \text { otherwise }\end{array}\right.$ then

$$
B\left(K_{m, n}, \mathbb{Z}_{k}\right) \geqslant \max \{m+f(k), n\}
$$

Proof. Suppose $k \mid m$ and $m \leqslant n \leqslant m+k-2$. Consider $f: E\left(K_{m+k-1, m+k-1}\right) \rightarrow$ $\mathbb{Z}_{k}$. Take $n$ vertices at one class of $K_{m+k-1, m+k-1}$ say $\left\{u_{1}, \ldots, u_{n}\right\}$ and all the $m+k-1$ vertices from the other class $\left\{w_{1}, \ldots, w_{m+k-1}\right\}$ Define $a_{i}=\sum_{j=1}^{n} f\left(w_{i}, u_{j}\right)$, $1 \leqslant i \leqslant m+k-1$. By theorem $A$ there exists $I \subset\{1,2, \ldots, m+k-1\},|I|=m$ such that $\sum_{i \in I} a_{i} \equiv 0(\bmod k)$. Clearly $\left\{u_{i}\right\}_{i=1}^{n}$ and $\left\{w_{i}, i \in I\right\}$ form a zero-sum copy ( $\bmod k)$ of $K_{m, n}$. The two other cases follow easily along the same line, proving (i). For (ii) consider the following $\mathbb{Z}_{k}$-coloring.

'Take a copy of $K_{m+f(k)-1, m+f(k)-1}$ with classes $\left\{u_{1}, \ldots, u_{m+f(k)-1}\right\}$ and $\left\{w_{1}, \ldots\right.$,

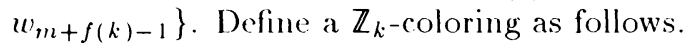

$$
f\left(u_{i}, u_{j}\right)= \begin{cases}1 & \text { iff } i \geqslant m \text { and } j \geqslant m \\ 0 & \text { otherwise. }\end{cases}
$$

Any copy of $K_{m, n}$ must contain some of the $u_{i}, i \geqslant m$, say a of them and some of the $w_{j}, j \geqslant m$, say $b$ of them.

For such a copy we have $\sum_{e \in E\left(K_{m, n}\right)} f(e)=a b \not \equiv 0(\bmod k)$ because of the definition of $f(k)$, and the fact that $a, b \leqslant f(k)$. Hence we must have $B\left(K_{m, n}, \mathbb{Z}_{k}\right) \geqslant \max \{m+$ $f(k), n\}$.

An immediate corollary of Theorem 3 is:

Theorem 4. Let $n \geqslant m \geqslant 1$ be integers and $k$ be a prime. Then

$$
B\left(K_{m, n}, \mathbb{Z}_{k}\right)= \begin{cases}m+k-1 & \text { if } k \mid m \quad m \leqslant n \leqslant m+k-2 . \\ n & \text { if } k \mid m \quad n \geqslant m+k-1 \\ & \text { (holds even if } k \text { is not a prime). }\end{cases}
$$

Remark. The main consequence of Theorem 3 is that if $k \mid m n$ and $k \leqslant$ $\max \{m, n\}$ then $B\left(K_{m, n}^{\cdot}, \mathbb{Z}_{k}\right)$ is small. So it is inevitable to ask what if $k \mid m n$ but $k>\max \{m, n\}$. Moreover even after Theorems 3 and 4 we have not yet determined $B\left(K_{1, n}, \mathbb{Z}_{k}\right)$ although we know that it is at most $n+k-1$. We shall take a closer look at these problems. 
Let's first derive a lower bound for $B\left(K_{m, n}, \mathbb{Z}_{k}\right)$ for large $k$.

Theorem 5. Suppose $k \mid n^{2}$ and further $n^{2} / k=t$ where $t$ is a fixed integer. Then $B\left(K_{n, n}, \mathbb{Z}_{k}\right) \geqslant \frac{n}{2 e} e^{n / 4 t^{2}}$.

Proof. We apply the "second moment" probabilistic argument.

Let $f: E\left(K_{m, m}\right) \rightarrow \mathbb{Z}_{k}$ be a random mapping, ( $m$ to be determined later), given by the rule

$$
f(e)= \begin{cases}1 & \text { with probability } \frac{k}{2 n^{2}}=\frac{1}{2 t} \\ 0 & \text { with probability } 1-\frac{1}{2 t} .\end{cases}
$$

For every copy of $K_{n, n}$ in $K_{m, m}$ let $Y=\sum_{e \in E\left(K_{n, n}\right)} f(e)$ be the edge-sum random variable. Then $Y \sim B\left(n^{2}, \frac{k}{2 n^{2}}\right), E(Y)=n^{2} \cdot \frac{k}{2 n^{2}}=\frac{k}{2}$ and $\sigma(Y)=\sqrt{n^{2} \frac{k}{2 n^{2}}\left(1-\frac{k}{2 n^{2}}\right)}<$ $\sqrt{\frac{k}{2}},(Y$ is a binomial random variable). By the standard approximation of the binomial distribution (see e.g. [BOL2] p. 11-12) the probability that $Y \equiv 0(\bmod k)$ (i.e., will deviate by at least $\sqrt{\frac{k}{2}}$ standard deviations from its expectation) is

$$
\leqslant \text { Prob }\left(|Y-E(Y)| \geqslant \frac{k}{2}\right) \leqslant 2 e^{-2 k^{2} / 4 n^{2}}=2 e^{-k^{2} / 2 n^{2}} \text {. }
$$

Hence if we choose $m$, such that $\left(\begin{array}{c}m \\ n\end{array}\right)^{2}<\frac{1}{2} e^{k^{2} / 2 n^{2}}$ then we infer that $B\left(K_{n, n}, \mathbb{Z}_{k}\right)>m$.

A simple calculation gives $m \leqslant \frac{n}{2 e} e^{k^{2} / 4 n^{3}}=\frac{n}{2 e} e^{n / 4 t^{2}}$. Hence $B\left(K_{n, n}, \mathbb{Z}_{k}\right) \geqslant$ $\frac{n}{2 e} e^{n / 4 t^{2}}$.

$R$ e $\mathrm{m}$ a r $\mathrm{k}$. The same argument gives an exponential lower bound for $B\left(K_{n, n}, \mathbb{Z}_{k}\right)$ if $k \mid n^{2}$ and $k>n^{1.5+\varepsilon}, \varepsilon>0$ fixed.

Let's now derive an upper bound for $B\left(K_{m, n}, \mathbb{Z}_{m n}\right)$.

\section{Theorem 6.}

$$
B\left(K_{m, n}, \mathbb{Z}_{m n}\right) \leqslant \min \left\{(2 n-2)\left(\begin{array}{c}
2 m-1 \\
m
\end{array}\right)+1,(2 m-2)\left(\begin{array}{c}
2 n-1 \\
n
\end{array}\right)+1\right\}
$$

Proof. Set $1+(2 n-2)\left(\begin{array}{c}2 m-1 \\ m\end{array}\right)=q$ and let $f: E\left(K_{q, q}\right) \rightarrow \mathbb{Z}_{m n}$. Choose $2 m-1$ vertices $A=\left\{v_{1}, \ldots, v_{2 m-1}\right\}$ from one class of $K_{y, q}$, and let $B$ denote the set of vertices of the other class. By theorem $A$, for each $u \in B$ there is a subset $A_{u} \subset A$ such that $\left|A_{u}\right|=m$ and $\sum_{v \in A_{u}} f(u, v) \equiv 0(\bmod m)$.

But there are $\left(\begin{array}{c}2 m-1 \\ m\end{array}\right)$ subsets of cardinality $m$ of $A$, and $|B|=q=(2 n-2)\left(\begin{array}{c}2 m-1 \\ m\end{array}\right)+$ 1 , hence there are $2 n-1$ vertices of $B$, say $u_{1}, u_{2}, \ldots, u_{2 n-1}$ such that $A_{u_{1}}=A_{u_{2}}=$ $\ldots=A_{u_{2 n-1}}:=D,(D \subset A)$. For each $1 \leqslant i \leqslant 2 n-1$ put $a_{i}=\frac{1}{m} \sum_{v \in D} f\left(u_{i}, v\right)$ and observe that $a_{i}$ must we an integer for $1 \leqslant i \leqslant 2 n-1$. 
Apply theorem $A$ again on $\left\{a_{1}, \ldots, a_{2 n-1}\right\}$. Then there is a subset $I \in\{1,2, \ldots$, $2 n-1\},|I|=n$, such that $\sum_{i \in I} a_{i} \equiv 0(\bmod n)$.

Now the complete bipartite graph $K_{m, n}$ with classes $V_{1}=D$ and $V_{2}=\left\{u_{i}: i \in I\right\}$ is a zero-sum copy $(\bmod m n)$ of $K_{m, n}$.

Remark. A rough estimate gives $\frac{n}{2 e} e^{n / 4} \leqslant B\left(K_{n, n}, \mathbf{Z}_{n^{2}}\right) \leqslant n 4^{n}$, but by the trivial observation that $B\left(K_{n, n}, \mathbf{Z}_{n^{2}}\right) \geqslant B\left(K_{n, n}, 2\right)$, and by the standard probabilistic argument we can improve the lower bound to $B\left(K_{n, n}, \mathbb{Z}_{n^{2}}\right) \geqslant \frac{n}{2 e} 2^{n / 2} \geqslant \frac{n}{2 e} e^{n / 4}$. Also by standard probabilistic argument one can show $B\left(K_{n, n}, n^{2}\right) \geqslant \frac{1}{3 n} n^{n}$.

Hence $B\left(K_{n, n}, \mathbb{Z}_{n^{2}}\right) \lll B\left(K_{n, n}, n^{2}\right)$.

Our last result is the exact determination of $B\left(K_{1, n}, \mathbb{Z}_{k}\right)$ and $B\left(n K_{2}, \mathbb{Z}_{k}\right)$.

Theorem 7. Let $n \geqslant k \geqslant 2$ be integers such that $k \mid n$. Then

$$
B\left(n K_{2}, \mathbf{Z}_{k}\right)=B\left(K_{1, n}, \mathbf{Z}_{k}\right)=n+k-1 .
$$

Proof. Let $f: E\left(K_{n+k-1, n+k-1}\right) \rightarrow \mathbf{Z}_{k}$. Then trivially by Theorem A (as it contains both a copy of $K_{1, n+k-1}$ and a copy of $\left.(n+k-1) K_{2}\right)$ there is a zero-sum $(\bmod k)$ copy of both $K_{1, n}$ and $n K_{2}$. For the lower bound of $B\left(K_{1, n}, \mathbb{Z}_{k}\right)$ take a copy of $K_{n+k-2, n+k-2}$ with classes $\left\{u_{1}, u_{2}, \ldots, u_{n+k-2}\right\}$ and $\left\{w_{1}, \ldots, w_{n+k-2}\right\}$.

$$
\text { Define } f\left(u_{i}, w_{j}\right)= \begin{cases}1 & \text { if } 1 \leqslant i \leqslant n-1 \text { and } n \leqslant j \leqslant n+k-2 \\
0 & \begin{array}{l}
\text { or } 1 \leqslant j \leqslant n-1 \text { and } n \leqslant i \leqslant n+k-2 \\
\text { otherwise. }
\end{array}\end{cases}
$$

It is easily verified that there is no zero-sum copy of $K_{1, n}$. For the lower bound of $B\left(n K_{2}, \mathbb{Z}_{k}\right)$ take again a copy of $K_{n+k-2, n+k-2}$ with classes as before.

$$
\text { Define } f\left(u_{i}, w_{j}\right)= \begin{cases}1 & \text { if } n \leqslant i \leqslant n+k-2 \\ 0 & \text { otherwise. }\end{cases}
$$

Once again it is easy to see that for every copy of $n K_{2}, 1 \leqslant \sum_{e \in E\left(n K_{2}\right)} f(e) \leqslant k-1$, hence no zero-sum copy of $n K_{2}$ exists.

In closing we suggest some further problems and conjectures, whose solution may contribute to our understanding of the behavior of the zero-sum bipartite Ramsey numbers.

Problem 1. Determine $B\left(G, \mathbb{Z}_{2}\right)$ for every graph $G$ such that $2 \mid e(G)$, or at least if $G$ is connected.

Problem 2. Determine $B\left(K_{m, n}, \mathbf{Z}_{k}\right)$ for $k \mid m n$ and $k \leqslant \max \{m, n\}$. Recall that by Theorem 3 this is a moderate number. 
Problem 3. Is it true that $\lim _{n \rightarrow \infty} B\left(K_{n, n}, \mathbb{Z}_{n^{2}}\right) / B\left(K_{n, n}, 2\right)=1$ ?

Conjecture. (A. Biallostocki) For $n \geqslant 2 B\left(K_{2, n}, \mathbb{Z}_{2 n}\right) \leqslant 4 n-3$.

Observe that by theorem $G$ we only know that $B\left(K_{2, n}, \mathbf{Z}_{2 n}\right) \leqslant 6 n-5$.

Acknowledgement. I am indebted to Noga Alon and Arie Bialostocki for several helpful remarks. I am also indebted to the referee for his suggestions.

\section{References}

[AKLMRS] N. Alon, D. Kleitman, R. Lipton, R. Meshulam, M. Rabin, J. Spencer: Set systems with no unioon of cardinality 0 modulo $m$., Graphs and Combinatorics 7 (1991), 97-99.

[ALCA] N. Alon, Y. Caro: On three zero-sum Ramsey-type problems, to appear in Journal of Graph Theorey..

[BCR] A. Bialostocki, Y. Caro, Y. Roditty: On zero-sum Turan numbers, Ars Combinatoria 29A (1990), 117-127.

[BD1] A. Bialostocki, P. Dierker: Zero-sum Ramsey theorems, Congressus Numerantium 70 (1990), 119-130.

[DB2] A. Bialostocki, P. Dieker: Zero-sum Ramsey numbers-small graphs, Ars Combinatoria 29A (193-198).

[BD3] A. Bialostocki, P. Dierker: On the Erdös-Ginzburg-Ziv theorem and the Ramsey numbers for stars and matchings, Discrete Math. in press.

[BOL1] B. Bollobas: Extremal Graph Theory, Academic Press, New York, 1978.

[BOL2] B. Bollobas: Random Graphs, Academic Press, New York, 1985.

[CAR1] Y. Caro: On zero-sum Ramsey numbers-stars, Discrete Mathematics 104 (1992), 1-6.

[CAR2] Y. Caro: On $q$-divisible hypergraphs, Ars Combinatoria, in press.

[CAR3] Y. Caro: On zero-sum Turan numbers-stars and cycles, Ars Combinatoria 33 (1992), 193-198.

[CAR4] Y. Caro: On Zero-sum $\Delta$-systems and multiple copies of hypergraphs, Jour. of Graph Theory 15 (1991), 511-521.

[CAR5] Y. Caro: On zero-sum Ramsey numbers-Complete graphs, Quarterly J. of Mathematics (Oxford) 43 (1992), 175-181.

[EGZ] P. Erdös, A. Ginzburg, A. Ziv: Theorem in the additive number theory, Bull. Research Council Israel 10F (1961), 41-43.

[GRS] R. Graham, B. Rothschild, J. Spencer: Ramsey Theory, Second edition, John Wiley, New York, 1990.

[SCS1] L. Schrijver, P. D. Seymour: A simpler proof and a generalization of the zero-trees theorem, Jour. Combinatorial Theory, Ser. A 58 (1991), 301-305.

[SCS2] L. Schrijver, P. D. Seymour: Spanning trees of different weights, in "Polyhedral Combinatorics". DIMACS Series, Discrete Math. and Theoret. Comp. Sc. 1 (1990), (eds. W. Cook and P. D. Seymour) 281-288, AMS-ACM Publisher.

[STE] D. Steinberg: Computational Matrix Algebra, McGraw-Hill Book Company, 1974.

Author's address: Department of Mathematics, School of Education, University of Haifa-Oranim, Tivon, 36-910, Israel. 